\title{
Radiographic and MRI Assessment of the Thrower's Elbow
}

\author{
G. M. Powell ${ }^{1}$ - N. S. Murthy ${ }^{1}$ - A. C. Johnson ${ }^{1}$ (i)
}

Accepted: 24 March 2021 / Published online: 17 April 2021

(C) The Author(s) 2021

\begin{abstract}
Purpose of Review Throwing athletes are vulnerable to elbow injuries, especially in the medial elbow, related to high stress and valgus load in both acute and chronic settings as a result of this complex biomechanical action. This current review details the relevant anatomy and imaging features of common elbow pathology identified with radiographs and MRI in throwing athletes. Recent Findings Although elbow pathology in throwing athletes is well documented, advances in imaging technology and technique, particularly with MRI, have allowed for more detailed and accurate imaging description and diagnosis.

Summary Pathology of thrower's elbow occurs in predictable patterns and can be reliably identified radiologically. Clinical history and physical examination should guide radiologic evaluation initially with radiographs and followed by an MRI optimized to the clinical question. Constellation of clinical, physical, and radiologic assessments should be used to guide management.
\end{abstract}

Keywords Elbow $\cdot$ Thrower's elbow $\cdot$ Overhead throwing athlete $\cdot$ MRI

\section{Introduction}

Throwing athletes are particularly prone to elbow injuries and include baseball, softball, football, and javelin throwers. Baseball pitchers experience higher rotational and angular velocities compared to other throwing sports [1]. The throwing motion is a complex biomechanical action which is most extensive in baseball pitchers and is divided into six phases: windup, early cocking, late cocking, acceleration, deceleration, and follow through [2]. The elbow is exposed to high stress and valgus load during the later phases of throwing which can often exceed the inherent strength of medial stabilizers. Elbow injuries in throwing athletes are typically related to valgus load resulting in acute and chronic overuse injuries

This article is part of the Topical Collection on Injuries in Overhead Athletes

\section{A. C. Johnson}

Johnson.Adam@mayo.edu

G. M. Powell

powell.garret@mayo.edu

N. S. Murthy

murthy.naveen@mayo.edu

1 Department of Radiology, Mayo Clinic, 200 First St. SW, Rochester, MN 55905, USA
$[3,4]$. Preoperative radiologic evaluation with radiographs and subsequent magnetic resonance imaging (MRI) allow for accurate diagnoses which help guide management. The purpose of this manuscript is to review imaging features of common elbow pathology identified with radiographs and MRI in throwing athletes.

\section{Technique}

Radiographic evaluation of the elbow should routinely be performed prior to advanced imaging to assess for abnormalities including malalignment, fracture, arthrosis, osteochondral defects, osteocartilaginous bodies, joint effusion, and soft tissue calcification. Anteroposterior and lateral radiographs are performed with the elbow extended and the forearm supinated and the elbow flexed to $90^{\circ}$ with the forearm pronated, respectively. Tube angle is perpendicular to the image receptor with source to image distance of 48 inches and exposure parameters of $55 \mathrm{kVp}$ and $4 \mathrm{mAs}$. If clinically warranted, oblique, flexion, extension, gunslinger lateral, radial head, and cubital tunnel projections can also be obtained for a more sensitive evaluation. Occasionally, radiographic or fluoroscopic stress views are used to assess ligament integrity and joint instability. Stress views may be beneficial in the setting of high clinical suspicion of instability and an equivocal or nondiagnostic MRI. 
MRI evaluation of the elbow should be performed at field strengths no less than $1.5 \mathrm{~T}$; $3.0 \mathrm{~T}$ is preferred due to higher spatial resolution. Patients are either placed in the prone "superman" or supine position with the arm at the side (forearm supinated and elbow extended). The latter position allows for a more accurate assessment of the anterior bundle of the ulnar collateral ligament (UCL) which is taut in extension. Alternatively, elbow flexion would be preferred to interrogate the integrity of the posterior band of the UCL. Image quality and field homogeneity are optimized in the "superman" position as the elbow is closer to the isocenter of the magnet. Phased array surface and circumferential coils are used to obtain an appropriate signal to noise ratio and adequate signal from the tissue volume, respectively [5-7].

Comprehensive multiplanar evaluation of the elbow includes T1-weighted or proton density and fluid-sensitive sequences, with at least one plane of T1-weighting, acquired in the axial, coronal, and sagittal planes. Inversion recovery sequences should replace fat-saturated fluid sensitive sequences to minimize magnetic field inhomogeneity in the setting of off-center positioning of the elbow relative to the magnet's isocenter [7].

High-resolution (512-384 × 256 matrix, 3-mm slice thickness) intermediate echo time fast spin echo (FSE) sequences are acquired in the coronal plane to reduce partial volume averaging and evaluate ligaments, tendons, and cartilage. The remainder of the elbow structures is thoroughly evaluated with high-resolution (512-384 × 256 matrix, 3-4-mm slice thickness) intermediate echo time FSE sequences acquired in the axial and sagittal planes. In skeletally immature patients, fat-suppressed gradient-recalled echo (GRE) is appropriate to characterize cartilage of unfused physes.

Although some authors prefer MR arthrography with intraarticular gadolinium-based contrast or saline to facilitate recognition of partial tears of closely apposed ligaments, in our experience, high-resolution noncontrast MRI is sufficient for diagnosing clinically relevant pathology [8-10]. Additionally, in the setting of MR arthrography, cartilage and synovial assessment can be confounded by adjacent hyperintense signal and joint capsule distention, respectively.

\section{Relevant Imaging Anatomy}

The UCL is comprised of anterior, posterior, and transverse bundles. The UCL serves as the primary restraint to valgus stress, particularly the anterior bundle. Originating on the undersurface of the medial epicondyle, the cord-like anterior band gently tapers and inserts within $2 \mathrm{~mm}$ of the sublime tubercle as it merges with the ulnar periosteum $[6,11,12]$. Although biomechanically the anterior bundle is divided into anterior and posterior bands, these are not typically appreciated as distinct structures on imaging [13-15]. The fan-shaped posterior bundle appears as a capsular thickening forming the floor of the cubital tunnel extending from the undersurface of the medial epicondyle to the margin of the trochlear notch $[16,17]$. The transverse bundle extends from the medial olecranon to the inferomedial coronoid process. The transverse bundle does not significantly contribute to valgus stability. As a result of routine MRI positioning of the elbow in extension, the posterior and transverse bundles are not routinely assessed.

The UCL is best evaluated in the coronal plane. Ligament morphology is assessed with GRE and FSE sequences, whereas signal intensity is assessed with inversion recovery and FSE sequences. Occasionally, the origin of the anterior bundle of the UCL can have a slightly striated appearance as a result of interdigitating fat; otherwise, the ligament is normally homogenously hypointense on all pulse sequences $[11,18]$. Deep to the origin of the posterior band of the anterior bundle, there is a prominent synovial fold which should not be interpreted as a tear $[3,8$, 19]. The UCL is in close apposition to the overlying deep muscle fibers of the flexor digitorum superficialis.

The flexor-pronator muscle complex originates from the medial epicondyle and is comprised of the pronator teres, flexor carpi radialis, palmaris longus, flexor digitorum superficialis, and flexor carpi ulnaris muscles with various distal attachments. Additionally, the flexor digitorum superficialis, flexor carpi ulnaris, pronator teres, and brachialis comprise the flexor-pronator mass [20]. This muscle group provides dynamic stability to valgus stress. Similar to the UCL, the flexor-pronator mass tendons are normally homogeneously hypointense on all pulse sequences. Like with ligaments, tendon morphology is assessed with GRE and FSE sequences, whereas signal intensity is assessed with inversion recovery and FSE sequences. The flexor-pronator mass is best evaluated in the axial and coronal planes.

The ulnar nerve courses through the arcade of Struthers approximately $8 \mathrm{~cm}$ proximal to the elbow, along the medial aspect of the distal triceps, passes posterior to the medial epicondyle, and through the cubital tunnel [21, 22]. The cubital tunnel floor is formed by the posterior bundle of the UCL and joint capsule, whereas the roof is formed proximally by the cubital tunnel retinaculum (Osborne ligament) and distally by the flexor carpi ulnaris aponeurosis (arcuate ligament) [23]. Occasionally, an anomalous anconeus epitrochlearis may overlie the cubital tunnel. The ulnar nerve commonly has no brachial branches and rarely provides innervation to the triceps [22]. Innervation to the flexor carpi ulnaris is typically via the first motor branches of the ulnar nerve as it courses between the muscular heads superficial to the anterior bundle of the UCL after exiting the cubital tunnel. Given the anatomic location of the ulnar nerve, it is exposed to traction forces related to valgus stress and flexion, as well as nerve compression $[21,23]$. 
The ulnar nerve is best evaluated in the axial plane. The isointense uniform fascicular morphology of the ulnar nerve is assessed with GRE and FSE sequences. The signal intensity of the ulnar nerve is normally isointense to slightly hyperintense when assessed with inversion recovery and FSE sequences. The size of the ulnar nerve should be maintained or gently taper as it courses about the elbow distally.

When evaluating musculoskeletal structures, it is imperative to understand technical limitations, which could be misdiagnosed as pathology. Although it is outside the scope of this article, recognition of imaging artifacts such as magic angle artifact is critical for interpretation. Magic angle artifact is most evident in sequences with a short echo time (TE) such as T1weighted, PD, and GRE sequences and is increasingly diminished with longer TE sequences such as T2-weighted sequences. Magic angle artifact results in an artefactual increase in signal intensity within highly organized tissue when it is oriented at approximately $55^{\circ}$ to the main magnetic field. Anatomic structures subject to this artifact include tendons, ligaments, and peripheral nerves. It is important not to misinterpret magic angle artifact as pathology when evaluating these structures.

\section{UCL Injuries}

As the primary static stabilizer of the medial elbow, the UCL provides $54 \%$ of the total resistance against valgus loading in flexion predominately relying on the anterior bundle between 30 and $120^{\circ}[24 \cdot]$. The posterior bundle of the UCL contributes to valgus resistance at elbow flexion angles greater than and equal to $120^{\circ}$ [25]. The elbow is flexed $30-90^{\circ}$ in the typical throwing motion which can generate valgus torque and medial tensile force that exceeds the inherent stability of the ligament; however, secondary stabilizers provide the necessary biomechanical support to prevent medial elbow failure $[24 \cdot, 25]$. Alternative methods of throwing (e.g., sidearm or submarine) and poor mechanics can lead to even greater forces across the ligament [24•].

Once the inherent strength is exceeded, ligamentous injury results. Acute injuries to the UCL can manifest as altered signal intensity, laxity, discontinuity, and reactive changes in the periligamentous soft tissues. Periligamentous edema with otherwise intact ligament fibers is consistent with a low-grade sprain in the appropriate clinical setting (Fig. 1) [23]. In the absence of a well-defined partial thickness tear, intrasubstance signal abnormality, ligament attenuation, and laxity represent interstitial microtears referred to as interstitial load [26].

Fiber discontinuity is consistent with tear. The UCL humeral origin is the most common location of a tear, whereas midsubstance and distal tears are least common [5]. Partial tears are classified as high grade or low grade based on greater or less than $50 \%$ involvement of the ligament thickness, respectively. Although partial thickness tears can be identified with fluid signal interposed between disrupted ligament fibers, more commonly they are diagnosed in the setting of ligament hyperintensity and indistinctness (Fig. 2) [26]. Additionally, a distal partial thickness tear can be identified when fluid insinuates between the deep fibers of the ligament which have stripped off from the sublime tubercle insertion [8]. The "Tsign" describes this appearance, which is seen with nonarthrographic and arthrographic MRI evaluations. Although the sensitivity of nonarthrographic MRI to evaluate partial thickness tears has been called into question, more recent studies have reported higher sensitivities when examining with high-resolution fluid sensitive intermediate echo time FSE sequences $[6,8,27]$.

A full thickness tear is present when there is complete fiber disruption which can be identified with a combination of

Fig. 1 Coronal T2 MR images of the elbow in different patients demonstrate periligamentous edema about the proximal (a, arrow) and distal (b, arrow) UCL consistent with low-grade sprains

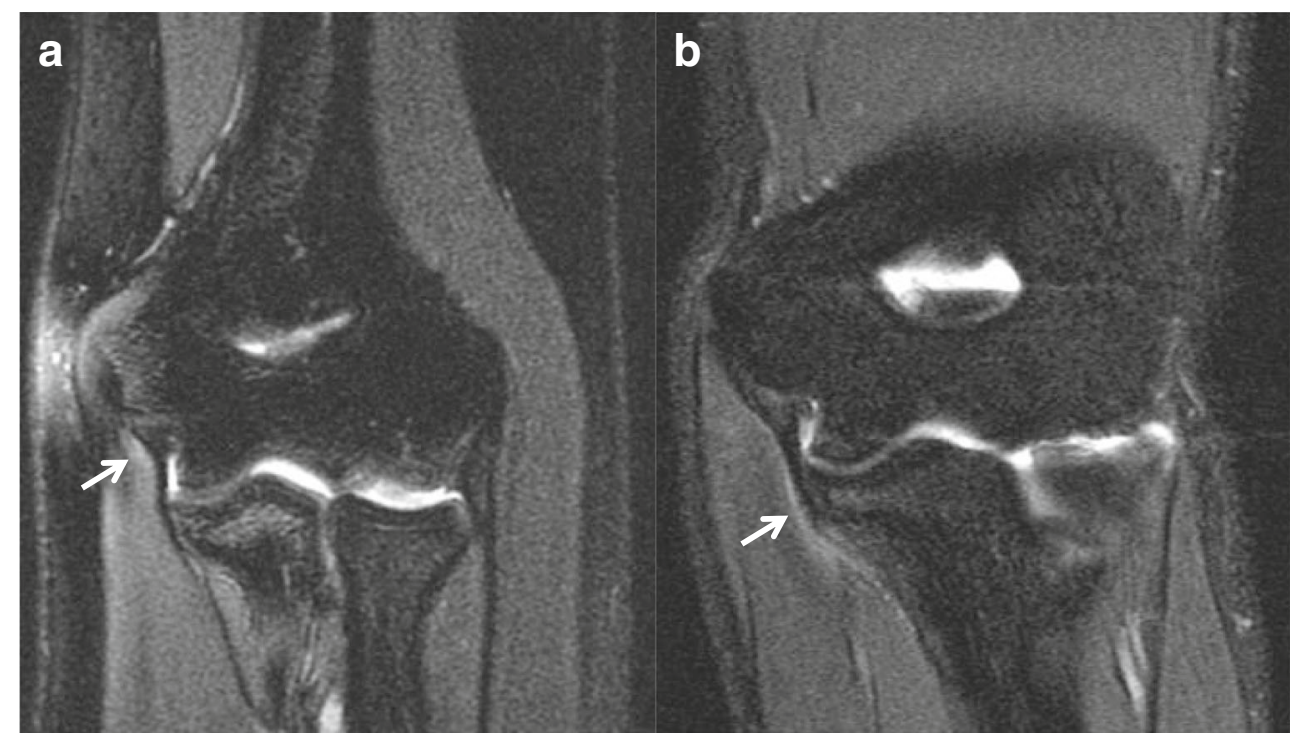


Fig. 2 Coronal T2 MR images of the elbow in different patients demonstrate a low- (a, arrow) and high-grade (b, arrow) partial thickness tears of the UCL

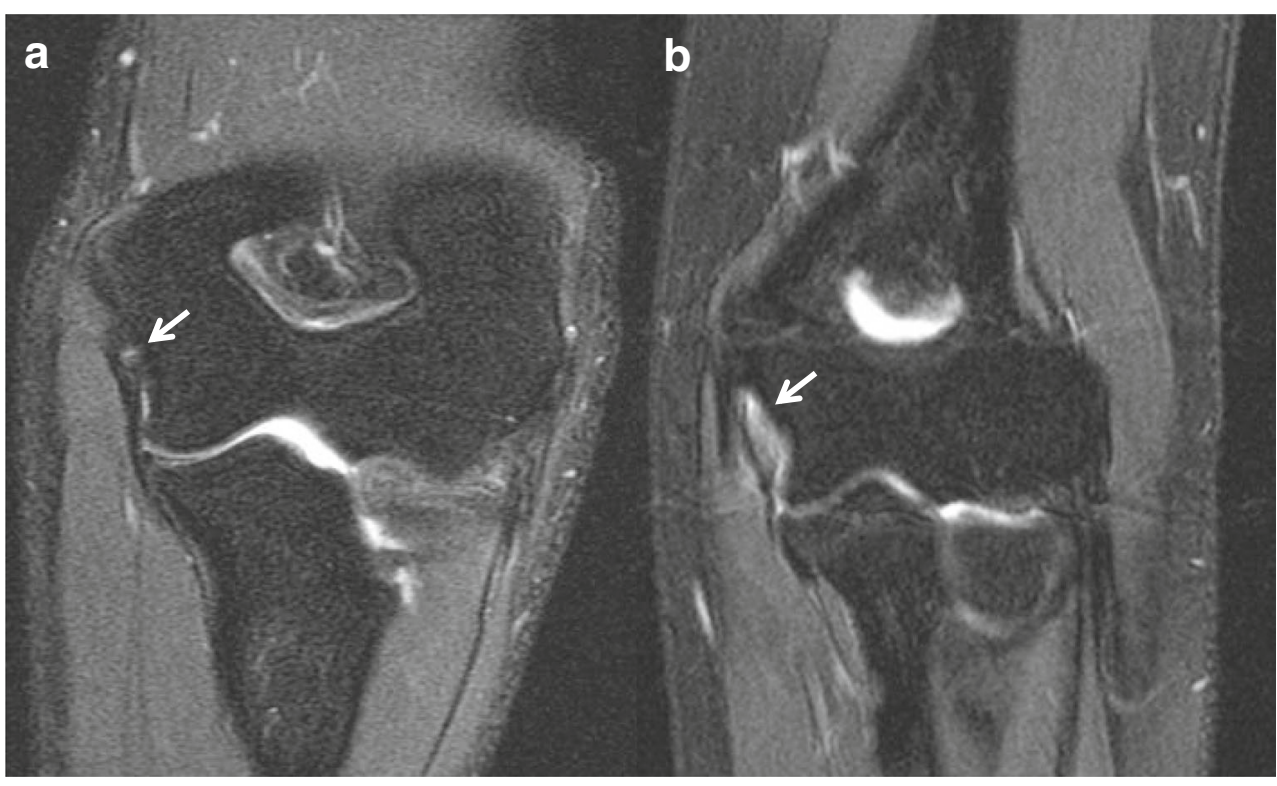

findings such as increased signal intensity and fiber indistinctness, laxity, or discontinuity in the absence of any distinctly intact fibers (Fig. 3). Additionally, an avulsion fracture of the sublime tubercle or traction osteophytes at the attachment of the UCL anterior bundle is functionally equivalent to complete disruption as this ligament no longer provides a functional valgus restraint to the medial elbow [27].

Ramkumar et al. have proposed a 6-stage MRI-based classification system for UCL injuries to aid in preoperative decision-making [28]. The classification system takes into consideration the location and degree of tear. The location of the tear is classified as proximal (1), midsubstance (2), or distal (3) UCL and degree of the tear as partial (A) or complete (B) (Fig. 4). Substantial to near perfect intraobserver and interobserver reliability has been demonstrated and this classification system can facilitate consideration of operative versus nonoperative management [28, 29].

Overuse and repetitive stress can result in chronic remodeling of the UCL. Even in the asymptomatic thrower, asymmetric ligament thickening and altered signal intensity have been identified (Fig. 5) [5, 30]. A chronically stressed UCL may also have mildly increased hyperintensity on fluid
Fig. 3 Coronal T2 MR images of the elbow in different patients demonstrate proximal (a, arrow) and distal (b, arrow) full thickness tears of the UCL

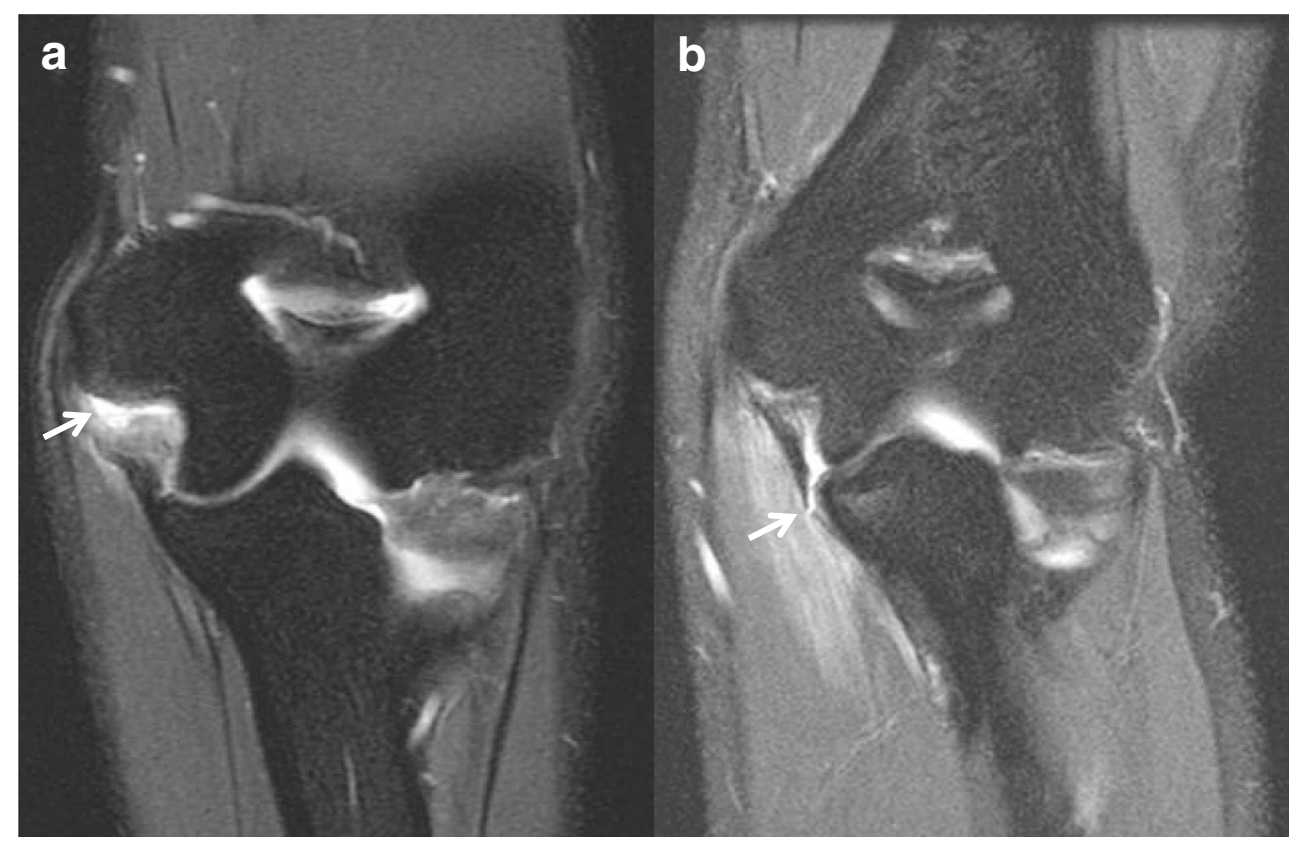




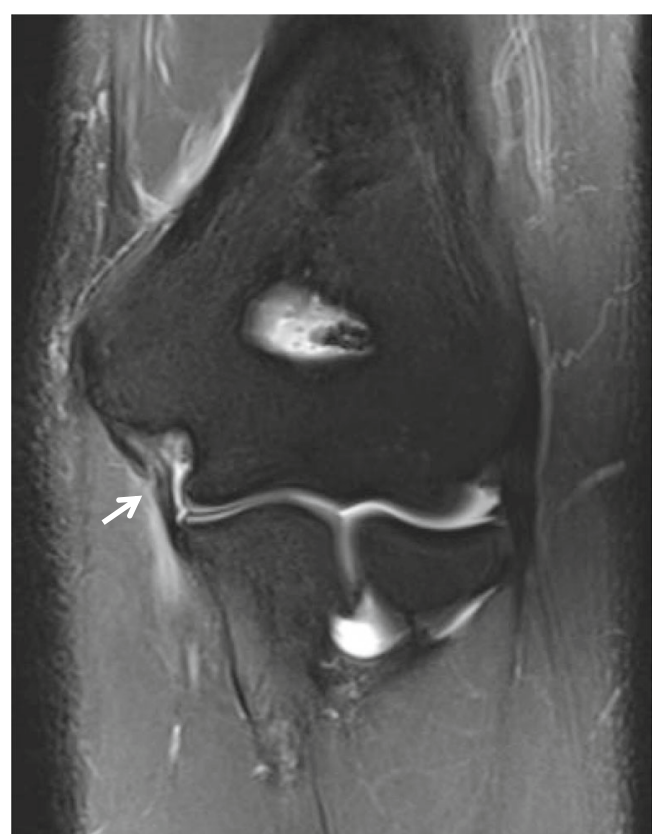

Fig. 4 Coronal T2 MR arthrogram of the elbow demonstrates a highgrade partial thickness tear of the UCL midsubstance consistent with a 2A injury (Ramkumar classification system, arrow)

sensitive sequences (representing chronic microtears), laxity, and/or redundancy [31-33]. Additionally, intraligamentous calcification and heterotopic ossification can be identified radiographically and with MRI representing sequelae of a remote or chronically injured UCL [26]. Reactive bone marrow edema-like signal at the UCL humeral or ulnar footprints can result from acute and chronic stress reaction related to traction.

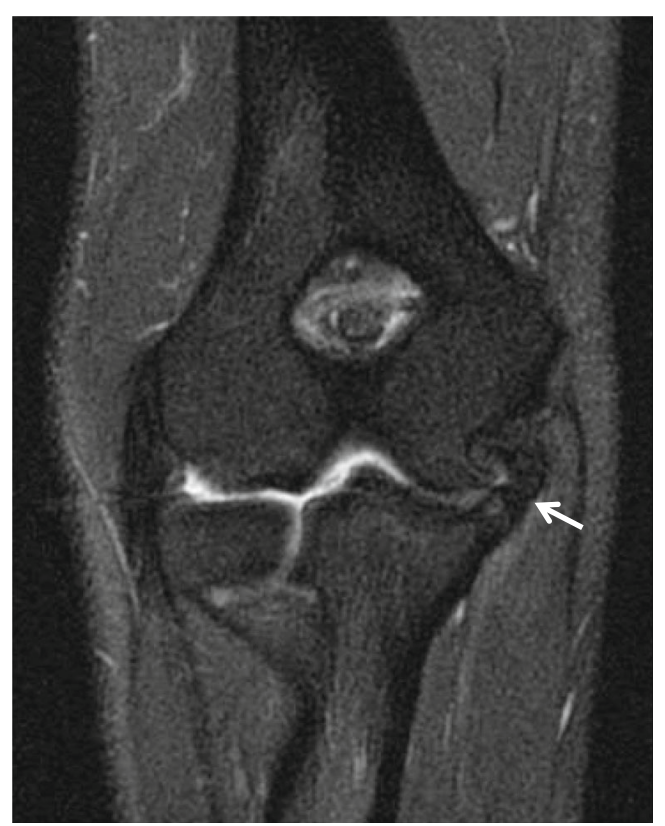

Fig. 5 Coronal T2 MR image of the elbow demonstrates chronic low signal thickening of the UCL (arrow)

\section{Chronic Valgus Overload}

During the late acceleration and follow-through phases of throwing, there is a large mechanical force and load on the posteromedial elbow $[22,25]$. Repetitive chronic valgus stress and overload can result in UCL attrition and, ultimately, failure [26]. Secondary posteromedial impingement and osteoarthrosis are most evident in the posteromedial joint space. Posteromedial synovitis as a result of posteromedial impingement is most conspicuous on sagittal and axial FSE sequence represented by synovial and capsular thickening and irregularity with or without pericapsular inflammation (Fig. 6). The associated osseous remodeling initially includes subchondral sclerosis particularly at the posteromedial ulna and abutting posterior trochlea. As posteromedial impingement progresses, chondral thinning in the posteromedial ulnohumeral articulation can lead to osteophyte formation especially on the posterior and medial olecranon [30]. Osteocartilaginous bodies can result from chondral injury and osteophyte fragmentation or fracture. Additional signs of posteromedial impingement as related to osteophyte formation or osteocartilaginous bodies can present with the inability to fully extend the elbow for standard image positioning.

\section{Medial Epicondylosis}

The flexor-pronator muscle complex provides dynamic stability to the medial elbow offloading the stress to the UCL [25]. This muscle group is activated in the acceleration phase of throwing and during forceful wrist flexion as the ball is released [21]. Specifically, the pronator teres and flexor carpi radialis are the most activated components during the acceleration phase and are also the most commonly injured in medial epicondylosis [3, 24•, 34]. Medial epicondylosis in the setting of chronic valgus overload manifests as tendinosis and tears. Intermediate to increased $\mathrm{T} 2$ signal intensity within the tendon substance corresponds to collagen disruption, tendon degeneration, and neovascularization with or without focal enlargement represents tendinosis [35]. Partial or full thickness tears demonstrate fluid signal between tendon fibers with associated fiber discontinuity and/or indistinctness (Fig. 7). In addition to these injuries, an acute valgus load to the elbow can result in contusion and extensive soft tissue edema [4]. Calcific tendinosis can also occur in the flexor-pronator complex manifesting as amorphous calcification at the medial elbow radiographically and an intratendinous hypointense nidus with adjacent edema on fluid sensitive sequences. Chronically degenerated or previously injured tendons can develop areas of heterotopic ossification or dystrophic calcifications. In the setting of medial epicondylosis, up to $60 \%$ of patients can have associated ulnar neuropathy [36]. 
Fig. 6 a Axial T1 MR image of the elbow demonstrates a small osteophyte along the posteromedial olecranon (arrow). b Axial T2 MR image demonstrates posteromedial ulnohumeral synovitis (arrow), mild pericapsular edema, and bone marrow edema-like signal in the olecranon

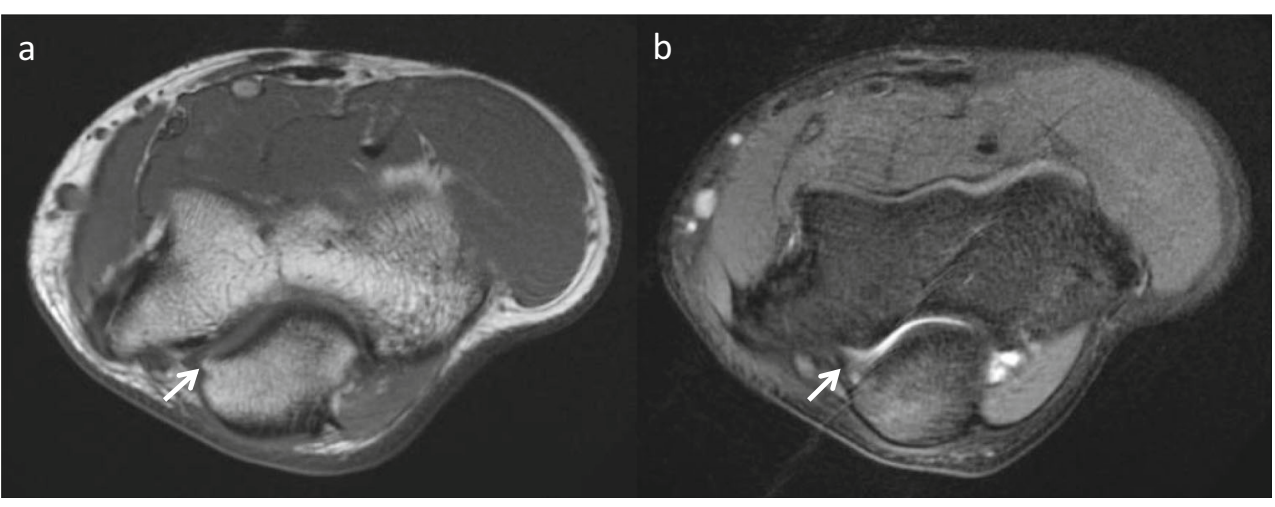

\section{Ulnar Neuropathy}

Ulnar neuropathy is common among throwing athletes as a result of concurrent medial elbow injury or in isolation [24•]. Types of injuries include traction, friction related to subluxation, and compression [25]. Chronic traction as a result of excessive valgus laxity is most common cause of ulnar nerve injury in throwing athletes $[21,26]$. Compression can occur at a number of sites and could be related to osteophytes, muscle hypertrophy, accessory and low lying muscles, fascial and ligamentous thickening, ganglia, and masses [23, 37].
Fig. 7 Coronal (a) and axial (b) T2 MR images of the elbow in the same patient demonstrate medial epicondylosis with low-grade partial thickness tears (arrow) and an adjacent UCL sprain. Coronal (c) and axial (d) T2 MR images of the elbow in a different patient demonstrate medial epicondylosis with high-grade partial thickness tears (arrow) and adjacent chronic thickening of the UCL

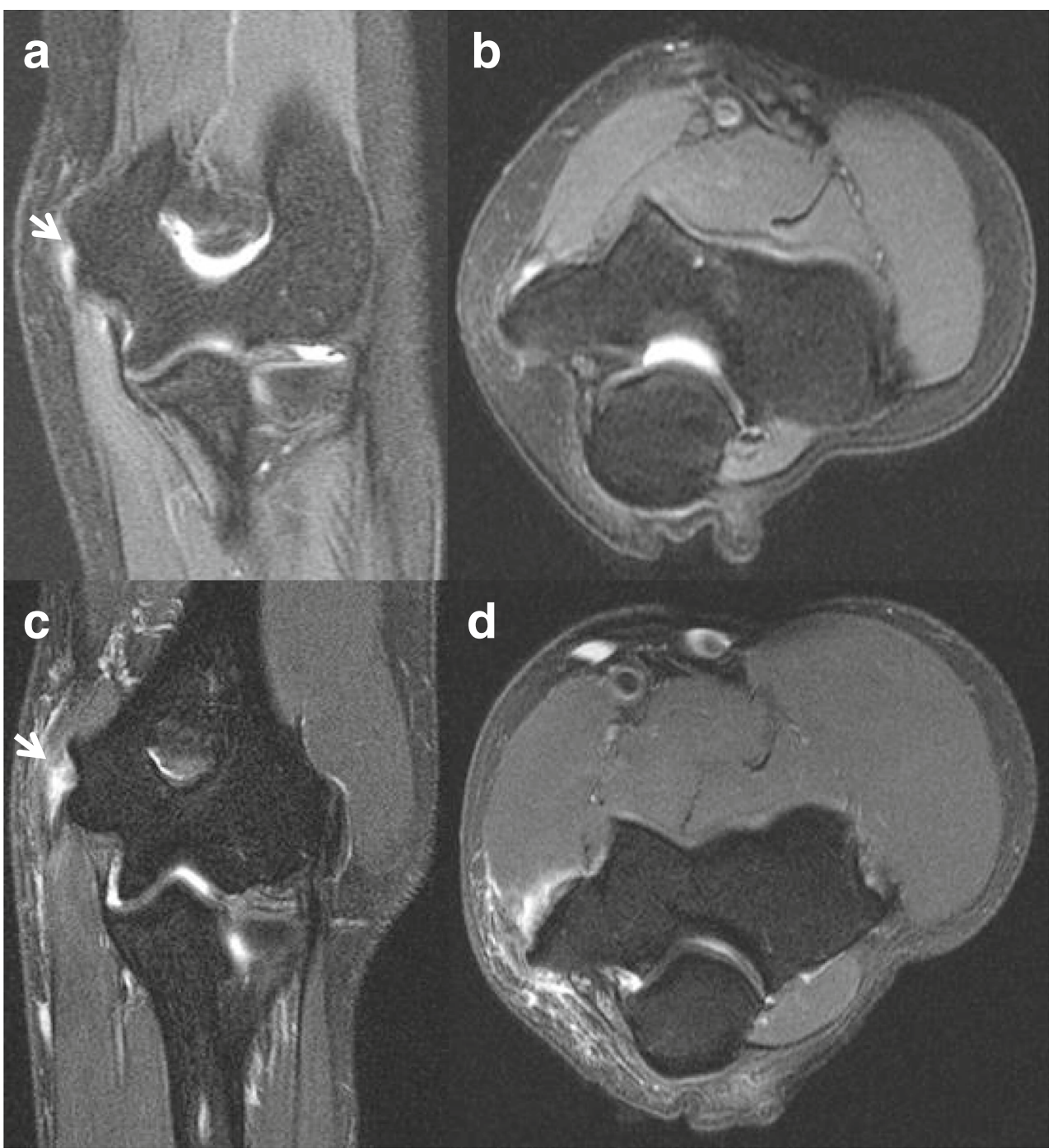




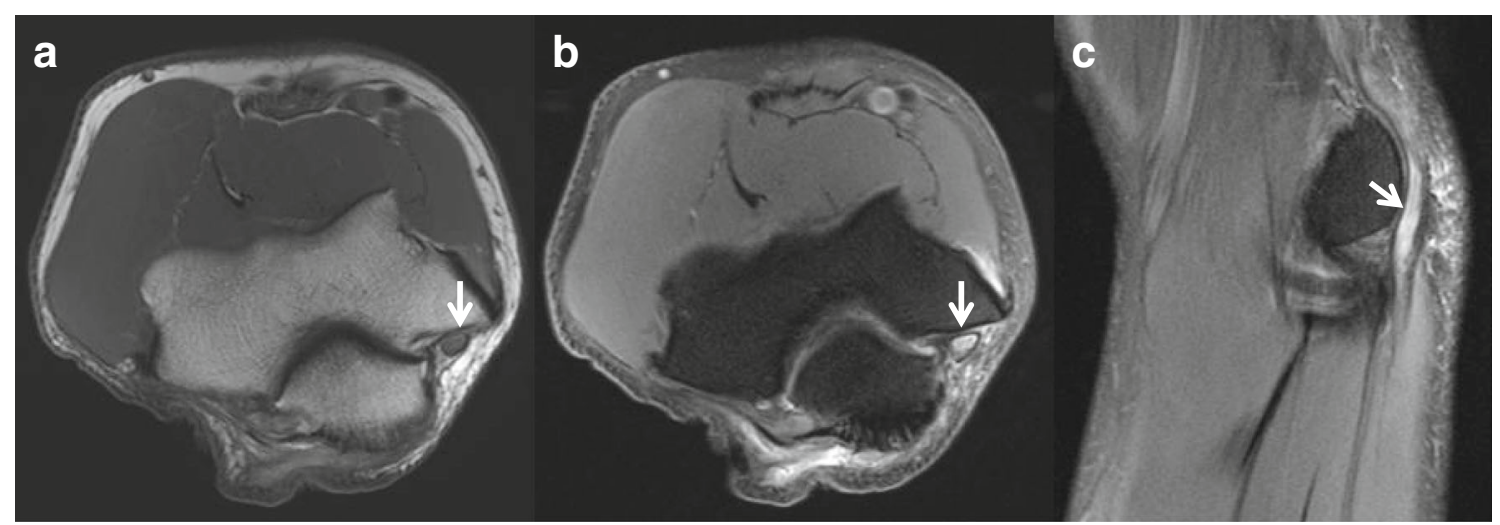

Fig. 8 Axial T1 (a) and axial (b) and coronal (c) T2 MR images of the elbow demonstrate ulnar nerve (arrows) enlargement, loss of the normal fascicular architecture, and T2 signal hyperintensity consistent with ulnar neuritis

Ulnar neuropathy may manifest as nerve or fascicular enlargement with or without loss of the normal fascicular architecture proximal to or within the cubital tunnel (Fig. 8). Hyperintense signal intensity can be identified on fluid sensitive sequences; however, this can also be seen in asymptomatic patients. Therefore, these findings are sensitive, but not specific for neuropathy and must be interpreted in the appropriate clinical setting [23].

\section{Osteochondral Lesions}

The etiology of osteochondral defects is multifactorial and primary osseous vascular insufficiency as a result of altered biomechanics and repetitive trauma with secondary involvement of the overlying cartilage is most likely [31, 38••]. Osteochondral injury can occur during an acute valgus load, direct impaction, or develop as a result of chronic valgus overload. Osteochondral lesions in throwing athletes occur most commonly in the capitellum; however, the radial head, trochlea, lateral trochlear ridge, olecranon, and olecranon fossa have also been reported [23, 39, 40]. Radiographically, osteochondral lesions can be identified as a curvilinear lucency undercutting subchondral bone and articular surface incongruity with or without osseous fragmentation and osteocartilaginous bodies (Fig. 9). Subtle subchondral flattening and mildly increased chondral hyperintensity on fluid sensitive sequences can represent evidence of an early osteochondral lesion; this was previously referred to as osteochondritis dissecans [41]. Additional osseous findings include a focal area of hazy subchondral $\mathrm{T} 1$ hypointensity and corresponding $\mathrm{T} 2$ hyperintensity or a more defined focal area of $\mathrm{T} 1$ hypointensity with a peripheral rim of $\mathrm{T} 2$ hyperintensity [39]. As these changes progress, there may be fragmentation, collapse, or cystic resorption of the subchondral bone plate. Lesions are also assessed for stability and an unstable fragment is indicated by fluid signal or cystic change interposed between the fragment and parent bone, sclerosis, fragmentation, and disruption of the overlying cartilage $[38 \bullet \bullet, 39]$. Various MRI staging systems exist for
Fig. 9 a Lateral radiograph of the elbow demonstrates osseous irregularity and sclerosis of the capitellum with subtle fragmentation (arrow). b Sagittal T2 MR image more clearly demonstrates collapse of the capitellar subchondral bone plate with subjacent cyst-like changes consistent with an osteochondral lesion

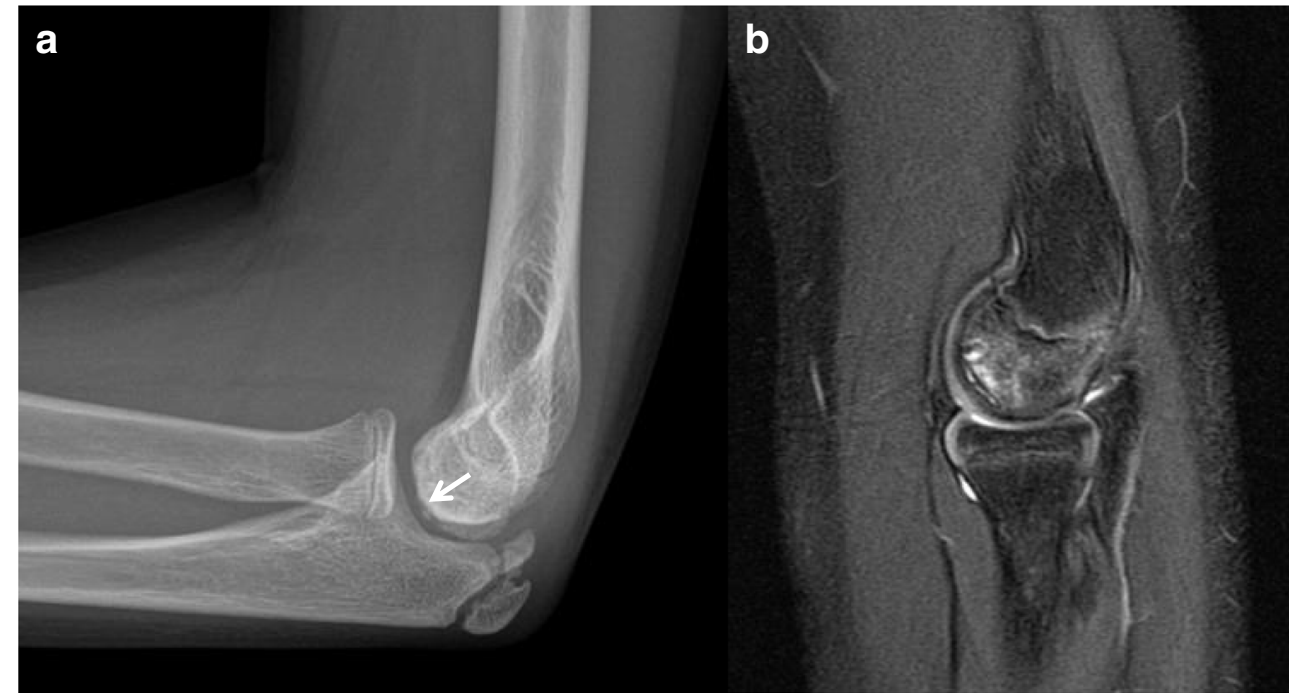


Fig. 10 a Anteroposterior radiograph of the elbow in a patient with prolonged pain demonstrates a bulbous medial epicondyle apophysis. b Axial T2 MR image demonstrates bone marrow edema-like signal in the medial epicondyle apophysis and increased signal in the physis with adjacent soft tissue edema.

Findings are consistent with medial epicondyle apophysitis

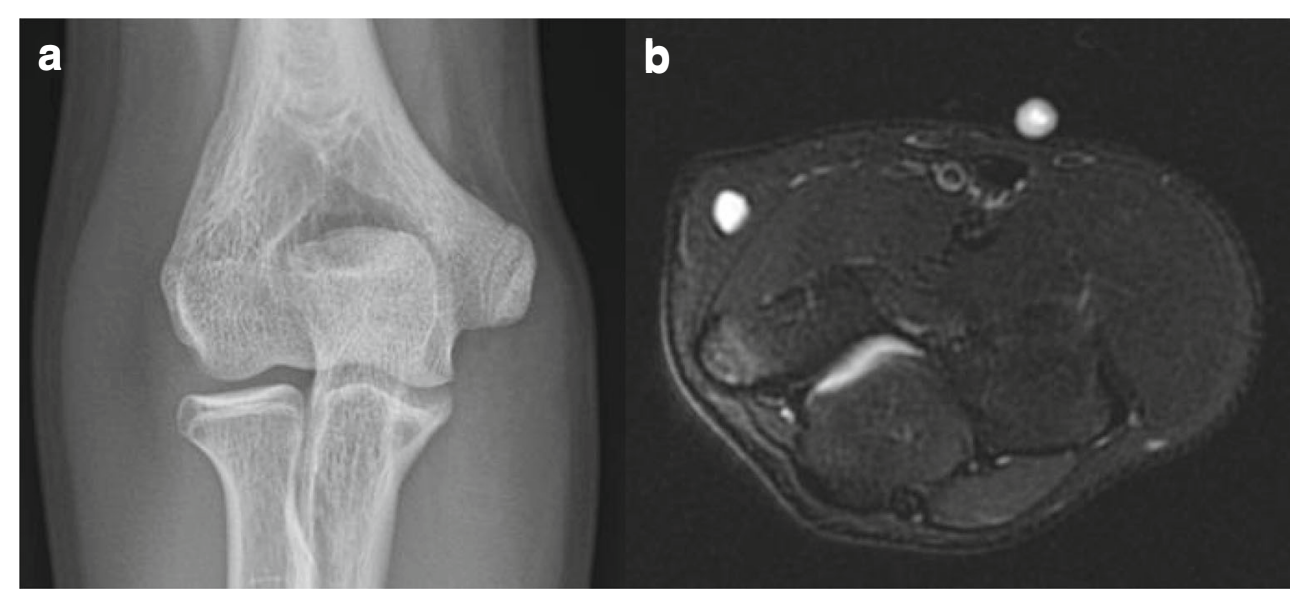

assessing the stability of osteochondral lesions in the elbow [42-44].

\section{Medial Epicondyle Apophysitis}

Acute and chronic stresses to the medial elbow in skeletally immature athletes are preferentially transmitted to the biomechanically weaker medial epicondylar apophysis rather than the UCL itself. This process is typically referred to as "little league elbow" $[25,45]$. In the acute setting, this may manifest as a physeal (Salter-Harris I) fracture with variable distraction, irregularity, and sclerosis [21, 25]. Physeal widening can also be seen in traction apophysitis with or without fragmentation of the medial epicondylar apophysis [5]. Associated bone marrow edema-like signal is easily identified on fluid sensitive sequences and can be seen with both injury patterns; therefore, differentiation is often dependent on clinical history (Fig. 10). Sequelae of a physeal injury prior to fusion may present as a more bulbous appearing medial epicondyle [26].

\section{Fracture}

Stress reaction and fracture in the throwing athlete's elbow can result from overuse and direct trauma. Oblique and transverse fractures involve the middle third of the olecranon as a result of repetitive olecranon fossa impaction from extension and valgus force, and extension force, respectively, with triceps traction during the deceleration phase of throwing [23,
Fig. 11 Axial T1 (a) and T2 (b) MR images of the elbow demonstrate an avulsed medial epicondyle apophysis (arrowheads) with distal distraction. There is also a lowgrade partial thickness tear of the proximal UCL (solid arrows)

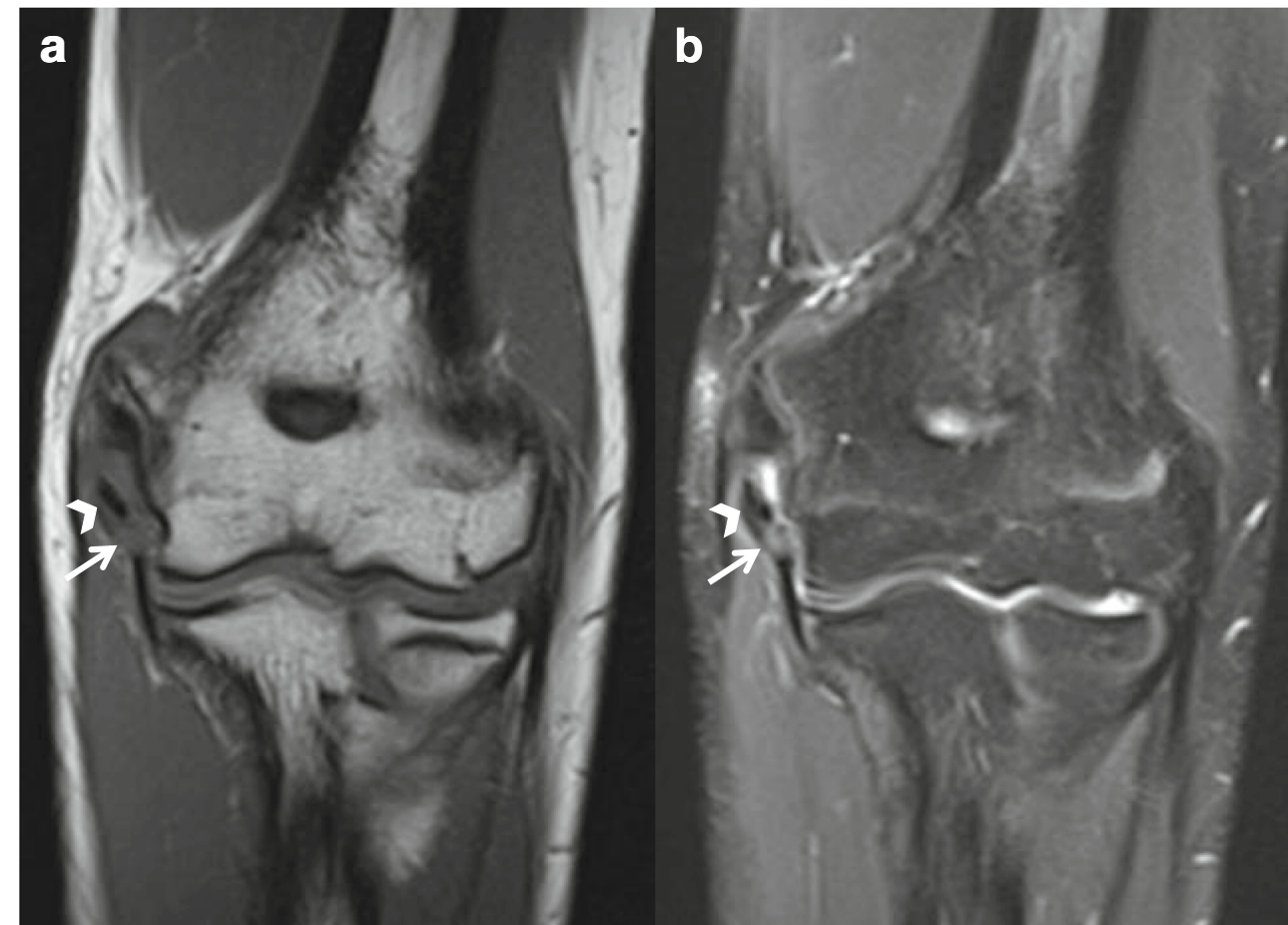


25]. Medial supracondylar stress fractures have also been described and are likely secondary to a similar process of impaction and valgus load [23]. If unfused physes are present, then traction apophysitis and physeal (Salter-Harris I) fracture can occur (Fig. 11) [25]. Stress reactions manifest as localized bone marrow edema-like signal and can evolve into distinct hypointense fracture lines. As previously described, an avulsion fracture of the sublime tubercle can result from valgus force to the elbow.

Chronic osseous remodeling and osteophyte formation at sites of ligamentous traction and articular degeneration are at risk for fracture and fragmentation in the acute and chronic setting. These fractured and fragmented osteophytes, in addition to acute and chronic chondral injury, can result in intraarticular osteocartilaginous bodies, which can result in further joint damage and progressive injury. Fractured osteophytes are commonly identified in the posterior joint space in the axial and/or coronal planes. Similarly, osteocartilaginous bodies can result from displacement or fragmentation of osteochondral defects. Although somewhat dependent on size, osteophytes and osteocartilaginous bodies can be identified with radiographs and MRI. Depending on the ratio of bone to cartilage, osteocartilaginous bodies can have a variable appearance on MRI, which ranges from fatty marrow to intermediate cartilage signal. Radiographically, osteophytes are best identified on the lateral radiograph with maximal flexion.

\section{Conclusion}

Predictable injury patterns occur in the thrower's elbow. Pathology is typically localized to the medial aspect of the joint and can be reliably identified with imaging. Clinical history and physical examination should guide imaging evaluation. Radiographs should be the first modality of choice used to identify malalignment, fracture, arthrosis, osteochondral defects, loose bodies, joint effusion, and soft tissue calcification. Subsequent evaluation with MRI provides superior soft tissue contrast for assessment of ligaments, muscle-tendon units, and nerves. The MRI technique should be optimized to maximize spatial resolution and soft tissue contrast. The constellation of clinical, physical, and radiologic examinations should be synthesized to guide management.

\section{Declarations}

Human and Animal Rights and Informed Consent This article does not contain any studies with human or animal subjects performed by any of the authors.

Conflict of Interest Garret Powell, Naveen Murthy, and Adam Johnson declare that they have no conflict of interest.
Open Access This article is licensed under a Creative Commons Attribution 4.0 International License, which permits use, sharing, adaptation, distribution and reproduction in any medium or format, as long as you give appropriate credit to the original author(s) and the source, provide a link to the Creative Commons licence, and indicate if changes were made. The images or other third party material in this article are included in the article's Creative Commons licence, unless indicated otherwise in a credit line to the material. If material is not included in the article's Creative Commons licence and your intended use is not permitted by statutory regulation or exceeds the permitted use, you will need to obtain permission directly from the copyright holder. To view a copy of this licence, visit http://creativecommons.org/licenses/by/4.0/.

\section{References}

Papers of particular interest, published recently, have been highlighted as:

- Of importance

•• Of major importance

1. Kirsch JM, Burrus MT, Bedi A. Common injuries in professional football quarterbacks. Curr Rev Musculoskelet Med. 2018;11:611.

2. Meister K. Injuries to the shoulder in the throwing athlete. Part one: biomechanics/pathophysiology/classification of injury. Am J Sports Med. 2000;28(2):265-75.

3. Kijowski R, Tuite M, Sanford M. Magnetic resonance imaging of the elbow. Part II: abnormalities of the ligaments, tendons, and nerves. Skelet Radiol. 2004;34(1):1-18.

4. Richard MJ. Traumatic valgus instability of the elbow: pathoanatomy and results of direct repair. J Bone Joint Surg Am. 2008;90(11):2416-22.

5. Potter HG, Ho ST, Altchek DW. Magnetic resonance imaging of the elbow. Semin Musculoskelet Radiol. 2004;8(1):5-16 (Copyright@ 2004 by Thieme Medical Publishers, Inc., 333 Seventh Avenue, New York, NY 10001, USA).

6. Kaplan LJ, Potter HG. MR imaging of ligament injuries to the elbow. Radiol Clin N Am. 2006;44(4):583-94.

7. Johnson D, Stevens KJ, Riley G, Shapiro L, Yoshioka H, Gold GE. Approach to MR imaging of the elbow and wrist: technical aspects and innovation. Magn Reson Imaging Clin N Am. 2015;23(3):35566.

8. Timmerman LAL, Schwartz MLM, Andrews JRJ. Preoperative evaluation of the ulnar collateral ligament by magnetic resonance imaging and computed tomography arthrography. Evaluation in 25 baseball players with surgical confirmation. Am J Sports Med. 1994;22(1):26-32.

9. Magee T. Accuracy of 3-T MR arthrography versus conventional 3T MRI of elbow tendons and ligaments compared with surgery. AJR Am J Roentgenol. 2015;204(1):W70-5.

10. LiMarzi GM, O’Dell MC, Scherer K, Pettis C, Wasyliw CW, Bancroft LW. Magnetic resonance arthrography of the wrist and elbow. Magn Reson Imaging Clin N Am. 2015;23(3):441-55.

11. Munshi M, Pretterklieber ML, Chung CB, Haghighi P, Cho JH, Trudell DJ, et al. Anterior bundle of ulnar collateral ligament: evaluation of anatomic relationships by using $\mathrm{mr}$ imaging, $\mathrm{mr}$ arthrography, and gross anatomic and histologic analysis. Radiology. 2004;231(3):797-803.

12. Sugimoto $H$, Ohsawa $T$. Ulnar collateral ligament in the growing elbow: MR imaging of normal development and throwing injuries. Radiology. 1994;192(2):417-22. 
13. Hotchkiss RN, Weiland AJ. Valgus stability of the elbow. J Orthop Res. 1987;5(3):372-7.

14. Callaway GH, Field LD, Deng XH, Torzilli PA, O'Brien SJ, Altchek DW, et al. Biomechanical evaluation of the medial collateral ligament of the elbow. J Bone Joint Surg Am. 1997;79(8): 1223-31.

15. Morrey BF, An K. Functional anatomy of the ligaments of the elbow. Clin Orthop Relat Res. 1985;201:84-90.

16. Bryce CDC, Armstrong ADA. Anatomy and biomechanics of the elbow. Orthop Clin North Am. 2008;39(2):141-54.

17. Hauptfleisch J, English C, Murphy D. Elbow magnetic resonance imaging: imaging anatomy and evaluation. Top Magn Reson Imaging. 2015;24(2):93-107.

18. Husarik DB, Saupe N, Pfirmann CWA, Jost B, Hodler J, Zanetti M. Ligaments and plicae of the elbow: normal $\mathrm{mr}$ imaging variability in 60 asymptomatic subjects. Radiology. 2010;257(1):185-94.

19. Gaary EA, Potter HG, Altchek DW. Medial elbow pain in the throwing athlete: MR imaging evaluation. AJR Am J Roentgenol. 1997;168(3):795-800 (PubMed-NCBI).

20. Cinque ME, Schickendantz M, Frangiamore S. Review of anatomy of the medial ulnar collateral ligament complex of the elbow. Curr Rev Musculoskelet Med. 2020;13:96-102.

21. Ouellette H, Kassarjian A, Tretreault P, Palmer W. Imaging of the overhead throwing athlete. Semin Musculoskelet Radiol. 2005;9(4):316-33.

22. McCarty LP 3rd. Approach to medial elbow pain in the throwing athlete. Curr Rev Musculoskelet Med. 2019;12(1):30-40.

23. Bucknor MD, Stevens KJ, Steinbach LS. Elbow imaging in sport: sports imaging series. Radiology. 2016;279(1):12-28.

24. Wong TT, Lin DJ, Ayyala RS, Kazam JK. Elbow injuries in adult overhead athletes. AJR Am J Roentgenol. 2017;208(3):W110-20 This article does good job detailing the MRI appearance and underlying biomechanical explanation of the findings related to posteromedial impingement.

25. Wo S, Mulcahy H, Richardson ML, Chew FS, Gee A, Hsu J, et al. Pathologies of the shoulder and elbow affecting the overhead throwing athlete. Skelet Radiol. 2017;46(7):873-88.

26. Lurie B, Fritz J, Potter H. MR imaging in patients with ulnar collateral ligament injury. In: Dines J, Altchek D, editors. Elbow ulnar collateral ligament injury. Boston: Springer; 2015.

27. Salvo JP, Rizio L, Zvijac JE, Uribe JW, Hechtman KS. Avulsion fracture of the ulnar sublime tubercle in overhead throwing athletes. Am J Sports Med. 2002;30(3):426-31.

28. Ramkumar PN, Frangiamore SJ, Navarro SM, Lynch TS, Forney MC, Kaar SG, et al. Interobserver and intraobserver reliability of an MRI-based classification system for injuries to the ulnar collateral ligament. Am J Sports Med. 2018;46(11):2755-60.

29. Ramkumar PN, Haeberle HS, Navarro SM, Frangiamore SJ, Farrow LD, Schickendantz MS. Prognostic utility of an magnetic resonance imaging-based classification for operative versus nonoperative management of ulnar collateral ligament tears: one-year follow-up. J Shoulder Elb Surg. 2019;28(6):1159-65.

30. Hurd WJ, Eby S, Kaufman KR, Murthy NS. Magnetic resonance imaging of the throwing elbow in the uninjured, high school-aged baseball pitcher. Am J Sports Med. 2011;39(4):722-8.
31. Potter HG. Imaging of posttraumatic and soft tissue dysfunction of the elbow. Clin Orthop Relat Res. 2000;2000(370):9-18.

32. Mirowitz SAS, London SLS. Ulnar collateral ligament injury in baseball pitchers: MR imaging evaluation. Radiology. 1992;185(2):573-6.

33. Dodson CC, Thomas A, Dines JS, Nho SJ, Williams RJ, Altchek DW. Medial ulnar collateral ligament reconstruction of the elbow in throwing athletes. Am J Sports Med. 2006;34(12):1926-32.

34. Glousman R, Barron J, Jobe FW, Perry J, Pink M. An electromyographic analysis of the elbow in normal and injured pitchers with medial collateral ligament insufficiency. Am J Sports Med. 1992;20:311-7.

35. Potter HGH, Hannafin JAJ, Morwessel RMR, DiCarlo EFE, O'Brien SJS, Altchek DWD. Lateral epicondylitis: correlation of MR imaging, surgical, and histopathologic findings. Radiology. 1995;196(1):43-6.

36. Gabel GT, Morrey BF. Operative treatment of medial epicondylitis: influence of concomitant ulnar neuropathy at the elbow. J Bone Joint Surg Am. 1995;77(7):1065-9.

37. Hash T, Bogner E. Nerve entrapment and compression syndromes of the elbow. Semin Musculoskelet Radiol. 2010;14(4):438-48.

38.• Logli AL, Bernard CD, O’Driscoll SW, et al. Osteochondritis dissecans lesions of the capitellum in overhead athletes: a review of current evidence and proposed treatment algorithm. Curr Rev Musculoskelet Med. 2019;12(1):1-12 An excellent review and discussion of the clinically important imaging findings of OCD lesions including their importance in directing treatment and predicting outcomes.

39. Collins MS, Tiegs-Heiden CA. Osteochondral lesions of the lateral trochlear ridge: a rare, subtle but important finding on advanced imaging in patients with elbow pain. Skelet Radiol. 2020;49(4): 593-9.

40. Patel NB, Thomas S, Lazarus ML. Throwing injuries of the upper extremity. Radiol Clin N Am. 2013;51(2):257-77.

41. Sofka CM, Potter HG. Imaging of elbow injuries in the child and adult athlete. Radiol Clin N Am. 2002;40(2):251-65.

42. Satake H, Takahara M, Harada M, Maruyama M. Preoperative imaging criteria for unstable osteochondritis dissecans of the capitellum. Clin Orthop Relat Res. 2013;471(4):1137-43.

43. Itsubo T, Murakami N, Uemura K, Nakamura K, Hayashi M, Uchiyama $\mathrm{S}$, et al. Magnetic resonance imaging staging to evaluate the stability of capitellar osteochondritis dissecans lesions. Am J Sports Med. 2009;37(2):298-304.

44. Kohyama S, Ogawa T, Mamizuka N, Hara Y, Yamazaki M. A magnetic resonance imaging-based staging system for osteochondritis dissecans of the elbow: a validation study against the International Cartilage Repair Society Classification. Orthop J Sports Med. 2018;6(9):2325967118794620.

45. Benjamin HJH, Briner WW Jr. Little league elbow. Clin J Sport Med. 2005;15(1):37-40.

Publisher's Note Springer Nature remains neutral with regard to jurisdictional claims in published maps and institutional affiliations. 